\title{
Dynamic Visual Acuity during Passive Head Thrusts in Canal Planes
}

\author{
Michael C. Schubert, ${ }^{1}$ Americo A. Migliaccio, ${ }^{1}$ and Charles C. Della Santina ${ }^{1,2}$ \\ ${ }^{1}$ Department of Otolaryngology/Head and Neck Surgery, Johns Hopkins School of Medicine, 601 N. Caroline St, \\ JHOC Rm. 6245, Baltimore, MD 21287-0910, USA \\ ${ }_{2}^{2}$ Department of Biomedical Engineering, Johns Hopkins School of Medicine, Baltimore, MD, USA
}

Received: 3 February 2006; Accepted: 19 May 2006; Online publication: 30 June 2006

\begin{abstract}
We sought to determine whether the dynamic visual acuity (DVA) test, which has been used to measure the function of the two horizontal semicircular canals (SCCs), could be adapted to measure the individual function of all six SCCs using transient, rapid, unpredictable head rotation stimuli (head thrusts) in the direction of maximum sensitivity of each SCC. We examined head-thrust DVA (htDVA) performance in 19 healthy control subjects, five patients before and six patients after plugging of one superior SCC for treatment of superior canal dehiscence, and two subjects with unilateral vestibular deafferentation (UVD) by vestibular neurectomy. We compared htDVA results for each SCC to vestibulo-ocular reflex gains measured using 3-D scleral coil recordings during a passive head-thrust-test paradigm. Individuals with normal vestibular function had similar htDVA scores for each of the six directions (canals) tested (mean $0.058 \pm 0.050 \mathrm{LogMAR}$ ). Individuals tested after surgical plugging of one superior SCC were similar to normal for all SCCs except the plugged SCC, which had significantly worse htDVA scores (mean $0.270 \pm 0.08$ LogMAR). Individuals with UVD had significantly worse htDVA scores for head rotations maximally exciting any of the ipsilesional SCC (mean $0.317 \pm 0.129 \log M A R$ ) and scores similar to normal subjects for contralesional rotations $(0.063 \pm 0.051 \mathrm{LogMAR})$. These findings suggest that the htDVA test, which does not require scleral coil
\end{abstract}

Correspondence to: Michael C. Schubert - Department of Otolaryngology/Head and Neck Surgery - Johns Hopkins School of Medicine 601 N. Caroline St, JHOC Rm. 6245, Baltimore, MD 21287-0910, USA. Telephone: +1-410-9553403; fax: +1-410-9556526; email: mschube1@jhmi.edu placement, magnetic field coils, or expensive oculography equipment, can provide a useful quantitative measure of individual SCC function.

Keywords: vestibular, semicircular canal planes, dynamic visual acuity, superior canal dehiscence

\section{INTRODUCTION}

The main function of the angular vestibulo-ocular reflex (VOR) is to stabilize images on each retina during head rotations. In individuals with a normal VOR, visual acuity during head movements typical of daily activities is similar to that measured with the head stationary. In contrast, individuals with vestibular hypofunction stabilize images on the retina poorly, resulting in a degradation of visual acuity during head movements that would normally excite the hypofunctional semicircular canals (SCCs) and corresponding pathways of the vestibular system. This change from static visual acuity to visual acuity during head movement can be quantified using the "dynamic visual acuity" (DVA) test, in which a subject's visual acuity is measured using optotype characters presented only while the head is moving within a certain range of rotational velocities (Herdman et al. 1998).

Typically, the DVA test stimulus involves a selfgenerated sinusoidal head rotation in the horizontal plane (i.e., about the axis of the horizontal SCCs) and is interpreted as a test of the horizontal SCCs as well as the neural pathways and extraocular muscles mediating the horizontal VOR. When tested using rapid horizontal head rotations in subjects with known unilateral labyrinthine hypofunction, the 
DVA test exhibits a marked asymmetry (Herdman et al. 1998) because the VOR depends mainly on input from the excited horizontal SCC, with the inhibited horizontal SCC contributing relatively little to the response (Ewald 1892; Aw et al. 1996). The superior and posterior SCC contributes little to the VOR during head rotation about the axis of the horizontal SCCs because they are approximately orthogonal to the horizontal SCC (Della Santina et al. 2005). The DVA test has therefore been proposed as a clinical test of individual horizontal SCC function.

Dynamic visual acuity has also been characterized for pitch head movements and for unpredictable, transient head rotations. Schubert et al. examined the utility of DVA using self-generated sinusoidal pitch head movements (i.e., about an interaural axis) in normal subjects and in patients with unilateral (UVH) or bilateral (BVH) vestibular hypofunction on caloric and horizontal rotary chair testing. They found that pitch DVA is specific $(90 \%$ for UVH and for BVH) but not very sensitive (23\% for UVH, $55 \%$ for $\mathrm{BVH}$ ) for detection of peripheral vestibular hypofunction (Schubert et al. 2002a). In addition to the fact that the intact labyrinth in subjects with UVH can mediate a pitch VOR, this poor sensitivity suggests that during the self-generated, sinusoidal head rotation employed in that study, patients may enlist mechanisms other than the VOR (such as preprogrammed saccades) to stabilize gaze during DVA (Schubert et al. 2002b).

Herdman et al. (2003) reported that horizontal DVA is worse when measured in UVH subjects during passive, unpredictable transient horizontal head rotations than during self-generated, predictable head movements. These findings parallel those found by 3-D scleral coil measurements of horizontal VOR gain using active and passive variations on the head-thrust-test paradigm (in which a subject attempts to fixate a spot $1.4 \mathrm{~m}$ in front of the nose during rapid, transient head rotations that are either delivered by the examiner or self-generated). Della Santina et al. (2002) showed that the apparent VOR gain for ipsilesional head rotations after unilateral labyrinthectomy is significantly lower for passive/ unpredictable stimuli than for self-generated transient or sinusoidal movements. Taken together, these studies suggest that although pitch DVA with selfgenerated sinusoidal head rotations is an insensitive measure of UVH, DVA tested during passive transient head-thrust rotations about individual SCC axes may provide a useful proxy for VOR gains measured by 3-D scleral coils or oculography.

The purpose of the present study was to measure individual SCC function using the DVA test during passive, transient, high-acceleration head rotations in the plane of the SCC being tested, then compare these scores with the corresponding VOR gain for each SCC measured using the "gold standard" 3-D scleral search coil technique. We sought to compare DVA scores and VOR gains in healthy individuals, subjects undergoing surgical plugging of a single SCC, and subjects who have undergone unilateral vestibular neurectomy. We hypothesized that DVA using head-thrust stimuli, which we refer to as "headthrust DVA" (htDVA), should correlate with VOR gains measured directly for each SCC using the scleral coil technique and should selectively reflect the function of each SCC within a given labyrinth. Given the ease, speed, and noninvasiveness of htDVA testing compared to traditional 3-D coils testing, identifying such a correlation should prompt consideration of using htDVA in the routine clinical and laboratory evaluation of SCC function.

\section{METHODS}

\section{Subjects}

We studied 19 normal subjects (mean age $34.4 \pm$ 11.8 years, range 19-61 years) that had no complaints of vertigo, dizziness, or imbalance and had normal DVA for active horizontal head rotation (Herdman et al. 1998). We also studied five subjects (mean age $47 \pm 9.1$ years, range 34-57 years) before and after plugging of one superior canal (SC) for treatment of superior canal dehiscence (SCD). The diagnosis of SCD was based on history of sound- and/or pressureinduced vertigo, physical exam revealing sound-or pressure-induced eye movements, and computed tomography in the plane of the affected canal (Minor 2005). An additional two subjects who had undergone unilateral vestibular nerve section (one for Ménière's disease and one for vestibular schwannoma) were also studied. Five normal subjects and all patient subjects underwent passive "head-thrust" VOR gain testing using the 3-D scleral search coil recording technique (Migliaccio et al. 2004). Participation in this study was voluntary, and all subjects consented to be a part of this project in accordance with a protocol approved by the Johns Hopkins School of Medicine Institutional Review Board.

\section{Scleral search coil technique}

Binocular eye movements were recorded in three rotational dimensions using a pair of search coils embedded in a silicone annulus placed on each eye. A search coil pair embedded in a bite block was used to measure head rotation. Eye and head angular positions were sampled at $500 \mathrm{~Hz}$ at 16-bit resolution. Analog signals were low-pass-filtered with a singlepole analog filter that had a 3-dB bandwidth of 
$100 \mathrm{~Hz}$. Digital (postsampled) signals were filtered with a 50-tap zero-phase low-pass digital finite impulse response filter with $50-\mathrm{Hz}$ bandwidth.

Each subject was tested while seated upright and centered within a uniform magnetic field, with the interpupillary line in the earth-horizontal plane. The subject's head was positioned so that Reid's line (from the superiormost point of the bony-cartilaginous junction of the external auditory canal to the lowest point of the cephalic edge of the infraorbital rim) was also in the earth-horizontal plane, which we term zero reference position.

\section{Head-thrust test}

A head thrust consists of a passive, unpredictable, manual head rotation with peak amplitude $\sim 20^{\circ}$, velocity $\sim 150^{\circ} / \mathrm{s}$, and acceleration $\sim 3000^{\circ} / \mathrm{s}^{2}$ (Halmagyi and Curthoys, 1988). Before the start of each head thrust, the subject's head was placed in the zero reference position for $5 \mathrm{~s}$, enabling eye and head angular position to be calibrated in vivo while the subject fixated a target light-emitting diode (LED), which was positioned directly in front of the subject at 124 $\mathrm{cm}$ along the naso-occipital axis. The room was completely dark except for this LED. Head thrusts were delivered manually in five planes: yaw (horizontal canal plane), left superior/anterior-right posterior canal plane (LARP), and right superior/anterior-left posterior canal plane (RALP).

\section{DVA test protocol}

Detailed descriptions of the DVA test have been reported previously (Herdman et al. 1998; Schubert et al. 2002a). In brief, subjects were seated $2 \mathrm{~m}$ directly in front of a high-resolution 18.1-viewableinch monitor with a refresh rate of $85 \mathrm{~Hz}$. Subjects who normally wore glasses or contact lenses for distant viewing were instructed to wear them during all DVA testing. Static visual acuity was measured first by repeatedly displaying a single optotype (the letter E, randomly rotated each trial by $0,90,180$, or $270^{\circ}$ ) on a computer monitor. Subjects viewed five optotypes per acuity level, with optotype size then being decremented in steps equivalent to a visual acuity change of $0.1 \operatorname{LogMAR}\left(\log _{10} X\right.$, where $X=$ the minimum angle resolved, in arcmin, with 1 arcmin $=1$ / $60^{\circ}$; Ferris et al. 1982). The better one's visual acuity, the lower one's LogMAR score, with $\operatorname{LogMAR}=-0.3$, $0,0.3,0.7,1.0$, and 1.3 corresponding to Snellen visual acuity of 20/10, 20/20, 20/40, 20/100, 20/200, and $20 / 400$, respectively.

Static visual acuity was first determined keeping the subject's head fixed. Static visual acuity was scored when the subject failed to correctly identify five optotypes on an acuity level or reached the LogMAR score of 0.000 (Snellen equivalency of 20/ 20 acuity). For the dynamic component of the test, a single-axis Watson rate sensor (Micromedical Tech-
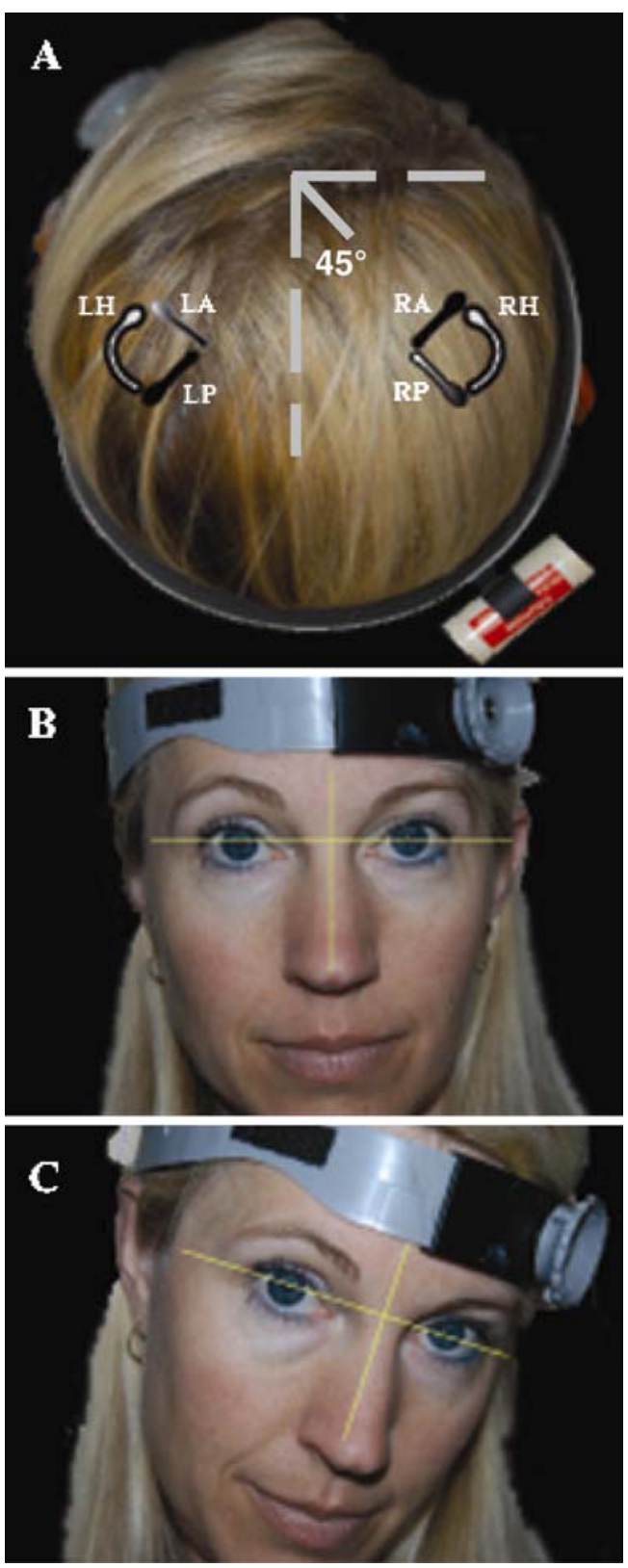

FIG. 1. Rate sensor and headband fit for htDVA test of left anterior and right posterior semicircular canal function. (A) The headband was placed $45^{\circ}$ relative to the midsagittal plane. The axis of maximum sensitivity of the rate sensor is aligned midway between the axes of the right posterior semicircular canal (RP) and left anterior semicircular canal (LA), which is approximately parallel. Each semicircular canal was tested independently, in its plane of excitation. The partially shaded semicircular canals illustrate the LARP plane. The black semicircular canals illustrate the right anterior (RA)/superior and left posterior (LP) canal plane (RALP). The right and left horizontal semicircular canals $(\mathrm{RH}, \mathrm{LH})$ are colored white. (B) Initial head position. (C) Final head position after the manually imposed head thrust. 
nologies, Inc., Chatham, IL, USA) was positioned on the subject's head so that the sensor's axis of maximum sensitivity approximately aligned with that of the SCC of interest (Della Santina et al. 2005). For example, when testing the LA and RP canal pair, the sensor was placed at $45^{\circ}$ right (subject's perspective) off the midsagittal line bisecting the skull, after pitching the head to bring Reid's line $\sim 20^{\circ}$ nose down (Fig. 1; Cremer et al. 1998; Della Santina et al. 2005). Horizontal head thrusts to assess horizontal canal function were performed first, followed by either RALP or LARP head thrusts. The direction of the head thrust was randomized within a given SCCpair plane. Normal subjects performed one practice trial for self-generated horizontal head rotations before we collected their htDVA score. These data were collected to compare DVA score with agematched controls (Herdman et al. 1998). One practice trial for a passive (manually imposed) horizontal head thrust was performed before commencing the htDVA test in the planes of the horizontal, superior, and posterior SCCs. During each head thrust, an optotype " $\mathrm{E}$ " randomly oriented in one of four directions was displayed on the monitor $2 \mathrm{~m}$ in front of the subject when head velocity, sensed by the Watson rate sensor, was between 120 and $180^{\circ}$ /s for more than $40 \mathrm{~ms}$. The optotype flashed on the monitor for no longer than $85 \mathrm{~ms}$, during which the head would have rotated $9-13.5^{\circ}$. To allow for blinks or transient loss of attention, the subject was allowed to view each optotype a maximum of three times, at which point the computer no longer displayed the letter and the subject was required to guess the orientation. Once the subject indicated a response, the next trial started. The test was terminated once the subject incorrectly identified all five optotype presentations at one acuity level (i.e., 20/ $\left.40_{-5}\right)$ or the subject reached the LogMAR of 0.000 (Snellen 20/20-0). The subscripted digit identifies the number of incorrect responses (five possible) at the acuity level listed.

The DVA test score was calculated by subtracting the static visual acuity LogMAR score from the DVA
LogMAR score. Additional information about LogMAR computation has been published elsewhere (Herdman et al. 1998; Schubert et al. 2002a).

\section{htDVA test program}

There are 11 visual acuity levels, five randomly oriented optotypes presented at each level, and three trials per optotype. In previous studies, the lowest acuity level was presented first and then increased sequentially, one level at a time-which meant that each test potentially required $165(11 \times 5 \times 3)$ subject responses. In this study, we modified the order in which the acuity levels were presented to reduce the number of subject responses. The htDVA test starts near the middle acuity level (level 6) and records the number of incorrect optotypes determined at each acuity level tested. Depending on the accuracy of the subjects' responses, the htDVA program advanced up or down to a new acuity level using a binary tree method. In pilot studies employing both techniques, this nonsequential method yielded exactly the same scores as the sequential method, but required only $\sim 100$ trials per subject. The time to complete the htDVA test was approximately $25 \mathrm{~min}$.

\section{Three-dimensional VOR gain data analysis}

Angular positions for eye and head with respect to space coordinates and eye with respect to head coordinates were represented by rotation vectors (Haslwanter 1995; Migliaccio and Todd 1999). Head-in-space, eye-in-space, and eye-in-head velocity vectors were calculated from the corresponding rotation vectors (Hepp 1990). Head velocity was calculated and reported with reference to a headfixed, right-handed coordinate frame (naso-occipital, interaural, and superior-inferior axes are parallel to the corresponding roll, pitch, and yaw axes, respectively), so that eye-in-head and head velocities were expressed with reference to exactly the same coordinate frame (Aw et al. 1996). Positive head roll, pitch,

TABLE 1

Normal and patient subjects tested

\begin{tabular}{lrlll}
\hline \multicolumn{1}{c}{ Diagnosis } & $N$ & \multicolumn{1}{c}{ Side of lesion } & \multicolumn{1}{c}{ Age (years) } & Use of corrective lenses \\
\hline Normal & 19 & n/a & $34.4 \pm 11.8$ & 9 total: 5 with contact lenses; 4 with glasses \\
SCD & 6 & & $47 \pm 9.1(34-57)$ & 6 \\
Before plugging & 5 & 2 bilateral, 2 right, 1 left & $46.8 \pm 9.5$ & 3 with contacts lenses, 1 with glasses \\
After plugging & 6 & 4 right, 2 left & $47.5 \pm 8.5$ & 2 with contacts lenses, 1 with glasses \\
UVD & 2 & 1 right, 1 left & $43 \pm 5.6(39$ and 47) & none \\
\hline
\end{tabular}

One additional subject was tested after SCD plugging.

$\mathrm{SCD}=$ superior canal dehiscence; UVD = unilateral vestibular deafferentation. 
TABLE 2

htDVA scores (LogMAR) for normal subjects and patients with unilateral vestibular deafferentation

\begin{tabular}{|c|c|c|c|c|c|c|}
\hline \multirow[b]{2}{*}{$I D$} & \multicolumn{2}{|c|}{ Horizontal canal } & \multicolumn{2}{|c|}{ Posterior canal } & \multicolumn{2}{|c|}{ Superior canal } \\
\hline & Right & Left & Right & Left & Right & Left \\
\hline Normal subjects & $\begin{array}{l}0.059 \pm 0.052 \\
\text { Ipsi }\end{array}$ & $\begin{array}{l}0.037 \pm 0.03 \\
\text { Contra }\end{array}$ & $\begin{array}{l}0.059 \pm 0.061 \\
\text { Ipsi }\end{array}$ & $\begin{array}{l}0.059 \pm 0.43 \\
\text { Contra }\end{array}$ & $\begin{array}{l}0.067 \pm 0.051 \\
\text { Ipsi }\end{array}$ & $\begin{array}{l}0.061 \pm 0.054 \\
\text { Contra }\end{array}$ \\
\hline UVD G & 0.328 & 0.031 & 0.564 & 0.071 & 0.321 & -0.02 \\
\hline UVD H & 0.222 & 0.076 & 0.247 & 0.112 & 0.222 & 0.112 \\
\hline
\end{tabular}

Normal LogMAR values reflect mean \pm 1 SD (ANOVA, $F=0.77, p=0.57$ ). Ipsi $=$ same side as unilateral vestibular deafferentation. Contra $=$ side opposite the affected canal. htDVA scores $>0.158$ are outside of the mean \pm 2 SD of LogMAR scores in normal subjects. Negative value indicates htDVA score better than static visual acuity.

and yaw velocities correspond to head rotations bringing the top of the head toward the right ear, the top of the head toward the nose, and the nose toward the left ear, respectively.

The time of onset of each head thrust was identified by fitting a polynomial curve to head-inspace velocity versus time. The time at which the magnitude of the fitted curve became greater than $2 \%$ of the curve's peak magnitude (typically, this threshold was $\sim 4^{\circ} / \mathrm{s}$ ) was defined as the time of onset. A similar approach was used to identify the time of onset of the eye movement responses. As the time between the onset of the head thrust and its maximum velocity was less than $150 \mathrm{~ms}$, analysis of the impulse data was restricted to a period of $150 \mathrm{~ms}$ from the onset. Trials of head impulse data that included blinks or in which the subject did not fix upon the target with both eyes at the onset of head rotation were not included in the analysis. Depending on the subject, approximately $10-20 \%$ of trials were rejected for this reason. The 3-D VOR gains, for head impulses delivered in all planes, were calculated by dividing each component of inverted eye velocity by the corresponding component of head velocity during the 30 -ms period prior to peak head velocity and averaged across trials.

Percent asymmetry values between ipsilesional and corresponding contralesional SCC VOR gains (i.e., ipsi- and contralesional horizontal canals) were calculated to determine the magnitude of VOR hypofunction after surgical lesion. We determined VOR asymmetry by subtracting mean contralesional from mean ipsilesional VOR gain values and then dividing by the sum of the mean ipsilesional and mean contralesional VOR gains.

\section{Statistical analysis}

All results are described as means \pm 1 standard deviation (SD). Two multiway analysis of variance tests were used to compare htDVA and VOR gain data each across the SCCs and different diagnostic groups. Post hoc analysis of variance (ANOVA) with
Tukey honestly significantly different (HSD) tests was used for multiple comparisons to control for a type I error. Post hoc paired $t$ tests were used when comparing data from the subjects with UVD. Linear regression was used to determine the variance in VOR gain that could be accounted for by the htDVA score. Pearson product moment correlation was used for comparing 3-D VOR gain and htDVA score. We used the mean plus two standard deviations of the htDVA scores obtained from the normal individuals as the reference criterion to define the "normal range" for htDVA scores.

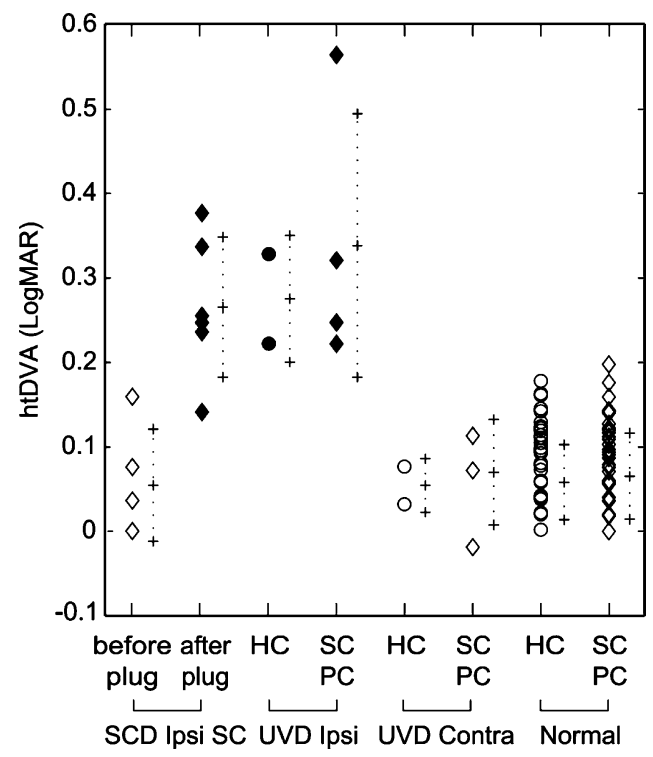

FIG. 2. Individual and mean \pm 1 SD error bars for htDVA score across diagnoses. The greater the htDVA score, the poorer the visual acuity during head thrusts. Values for SCD reflect those htDVA scores in the plane of the affected superior canal, before and after plugging. Values for normal reflect those htDVA scores from nonsurgically involved canals. Only those semicircular canals that were surgically lesioned were significantly different $(p<0.0001)$. $\mathrm{SCD}=$ superior canal dehiscence; $\mathrm{UVD}=$ unilateral vestibular deafferentation; $\mathrm{HC}=$ horizontal canal; $\mathrm{SC}=$ superior/anterior canal; $\mathrm{PC}=$ posterior/inferior canal; htDVA = head thrust dynamic visual acuity; $\log M A R=$ logarithm of the minimal angle resolvable. 


\section{RESULTS}

\section{htDVA Score}

We studied htDVA in canal planes for 19 healthy subjects, seven patients with SCD, and two patients with unilateral vestibular deafferentation (UVD) surgery. Of the seven patients with SCD, we examined the htDVA score for both pre- and postoperative SC plugging in four subjects. Sixteen (57\%) of the 28 subjects tested wore either glasses or contact lenses (Table 1).

Overall, we found a significant interaction between the htDVA score, the SCC tested, and the diagnostic group [multiple ANOVA (MANOVA), $F=$ $\left.8.3, p<10^{-12}\right]$. Post hoc testing revealed no difference in htDVA score across canal planes for normal subjects (ANOVA, $F=0.77, p=0.57$; see Table 2). As a result, we combined LogMAR score across the six canal planes for a mean htDVA score of $0.058 \pm$ 0.050 LogMAR. This is equivalent to incorrectly identifying less than three optotypes on the 20/20 line of a Snellen visual acuity chart. Next, we used the mean plus two standard deviations (0.158 LogMAR) as the criterion reference score to compare with htDVA scores of surgically plugged or denervated SCCs. Therefore, any htDVA score $>0.158$ LogMAR was considered abnormal.

Before canal plugging, individuals with SCD had no difference in htDVA scores for any of the six canals tested (ipsilesional or contralesional head rotations; ANOVA, $F=0.24, p=0.94$ ). However, after plugging of the affected SC, htDVA was significantly worse only in the plane of that canal-for every subject (Tukey HSD, $F=13.01, p<0.0001$ ), as illustrated by Figure 2. The mean htDVA score for individuals with one plugged SC was $0.271 \pm 0.091$ LogMAR. This mean is equivalent to missing four of five optotypes on the 20/30 line of a Snellen visual acuity chart.

For the two individuals tested after UVD via vestibular nerve section, we compared the mean difference between the ipsilesional canal htDVA

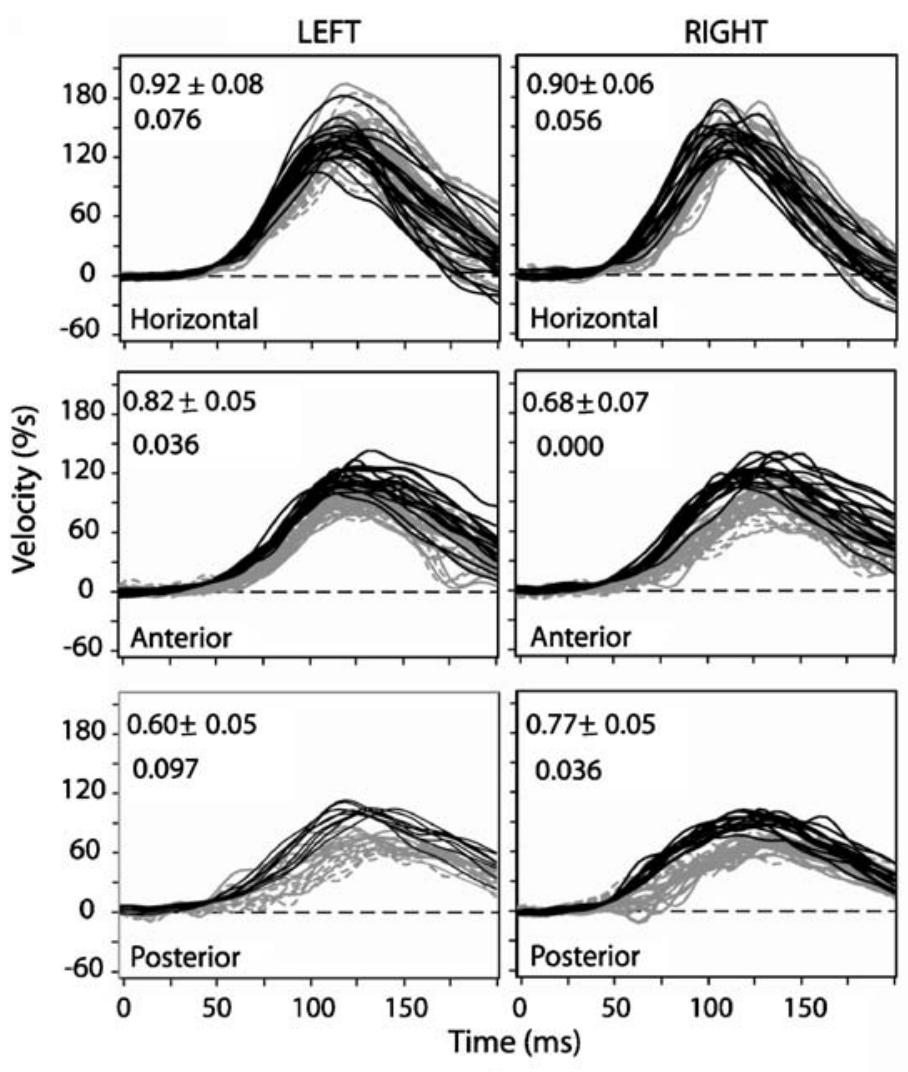

A

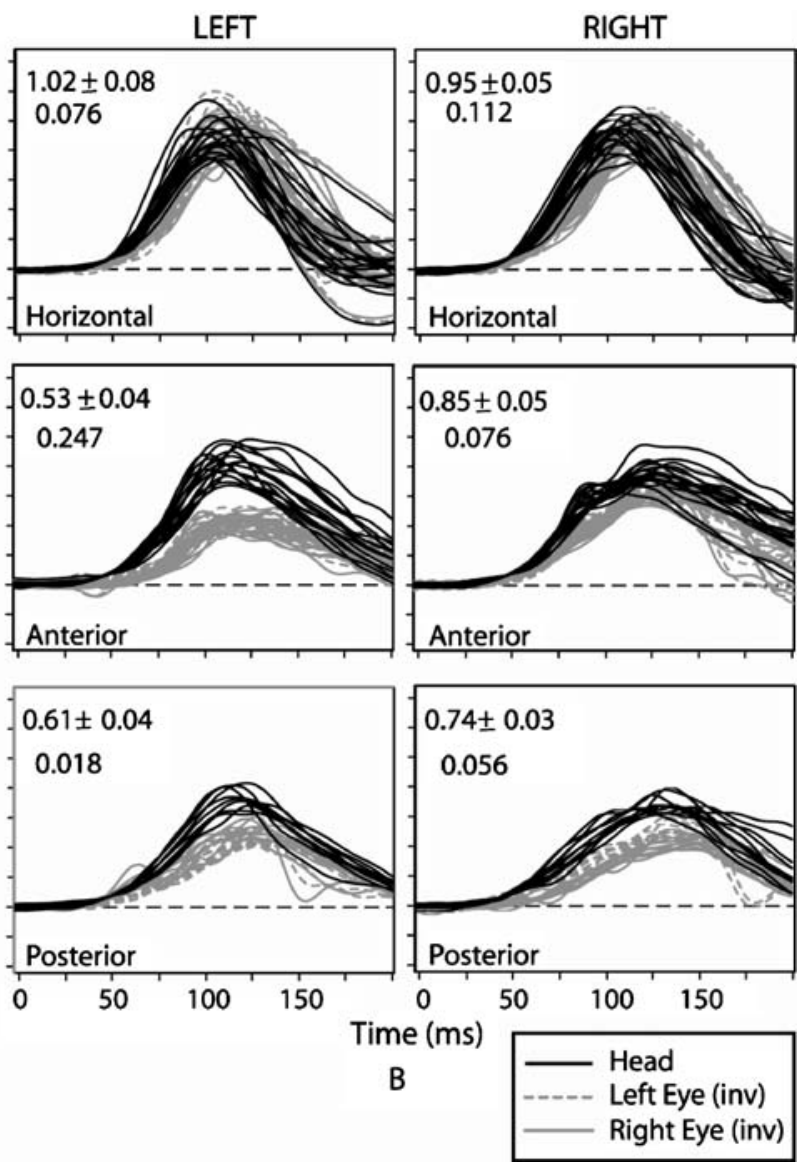

poorer the visual acuity during head thrusts. Panel (A) reflects the VOR gain and htDVA scores before superior canal plugging. Panel (B) reflects the VOR gain and htDVA scores after superior canal plugging. Eye velocity traces have been inverted for ease of comparison.
FIG. 3. Mean \pm 1 SD head and eye velocity traces with htDVA score before and after left superior canal plugging in one subject. 3-D VOR gain data collected in canal planes during passive headthrust testing in darkness. Smaller values in upper left corner reflect mean \pm 1 SD for 3-D VOR gain. Larger number beneath the 3-D VOR gain reflects the htDVA score. The greater the htDVA score, the 
scores $(0.317 \pm 0.129 \operatorname{LogMAR})$ and the contralesional canal htDVA scores $(0.063 \pm 0.05 \operatorname{LogMAR})$. The difference in htDVA score was significant (oneway paired $t$ test, $p=0.004$ ). Individual canal htDVA scores are listed in Table 2.

\section{Three-dimensional VOR gain}

We found a significant interaction between the 3-D VOR gain, the SCC tested, and the diagnostic group (MANOVA, $F=7.31, p<0.0001$ ). Figure 3 shows 3-D VOR gains and htDVA scores before and after superior SCC plugging surgery for one subject with SCD. Note that the htDVA score is above the criterion reference LogMAR score. For all subjects who had both htDVA and 3-D VOR gains determined for each of their SCC (five pre-SCD, six post-SCD, two UVD, four normal), we found a negative correlation between 3-D VOR gain and htDVA score (Pearson product moment, $r=-0.68)$, with htDVA score accounting for $45 \%$ of the variability in determining an abnormally low 3-D VOR gain (linear regression, $F=90.6, p=6.6 \times 10^{-15}$; Fig. 4 ).

Before SCD plugging, the mean asymmetry in VOR gain (ipsi - contra)/(ipsi + contra) between ipsilesional and contralesional SCCs was $1.0 \pm 2.2 \%$ for the horizontal, $3.0 \pm 8.8 \%$ for the posterior, and $1.1 \pm 9.4 \%$ for the SCs. The VOR gains from the vertical canals were $0.77 \pm 0.11$ (SC affected), $0.75 \pm$ 0.07 (SC unaffected), and $0.78 \pm 0.14$ (PC affected).

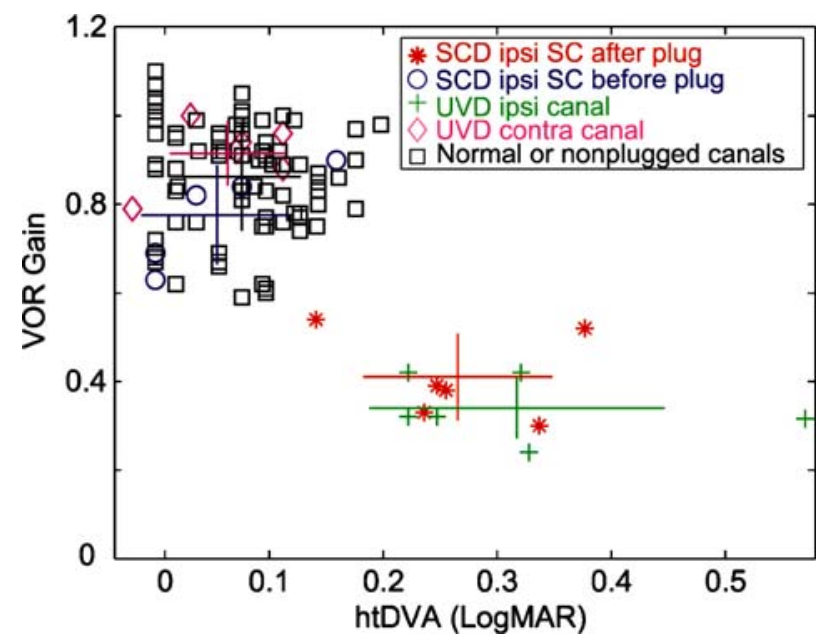

FIG. 4. Relationship between VOR gain and htDVA score. Individual 3-D VOR gain and htDVA scores are represented by symbols. Star and cross symbols reflect semicircular canals/vestibular nerves that have been surgically lesioned. Open symbols reflect nonlesioned semicircular canals/vestibular nerves. The horizontal lines represent $\pm 1 \mathrm{SD}$ for the htDVA score, whereas the vertical lines represent \pm 1 SD for the 3-D VOR gain. Colored lines match colored symbols. The point where the lines intersect is the mean of both htDVA and VOR gain values.

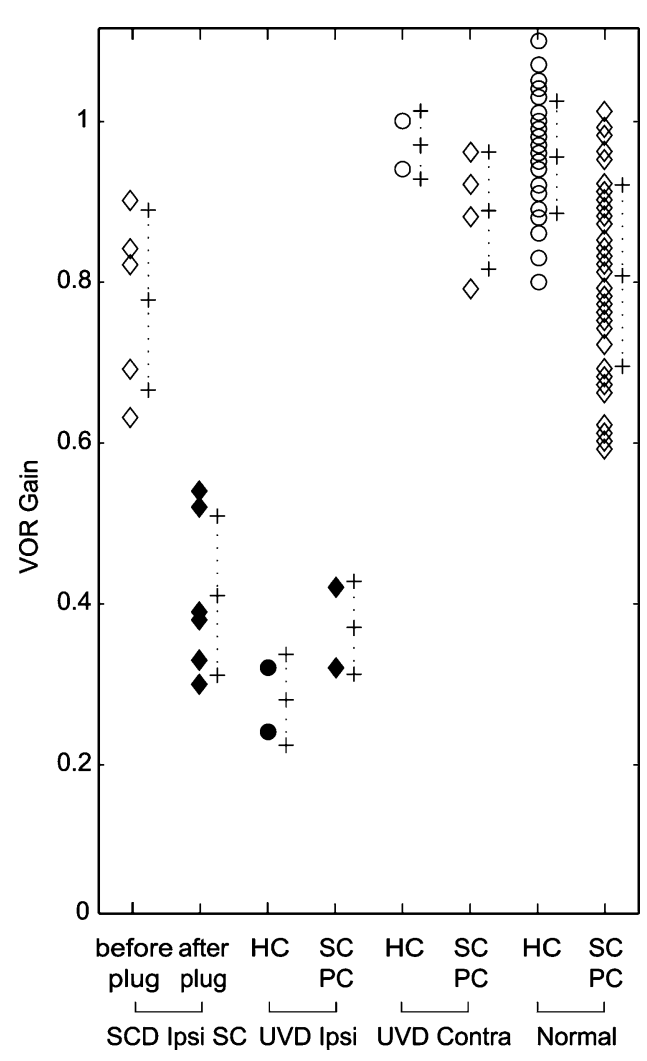

FIG. 5. Individual VOR gain with $1 \mathrm{SD}$ error bars across diagnoses. See Figure 2 for legend. VOR gain values for SCD ipsi SC reflect the affected superior canal, before and after plugging. Ipsi = same side as plugged SCD/UVD; contra = side opposite the affected canal/ lesioned nerve. Note that of the two subjects tested with UVD, the VOR gains for the ipsilesional SC and PC canals were the same as those gains illustrated (0.32 and 0.42$)$ and are therefore not represented.

These values were significantly less than the horizontal canals (HC affected $0.96 \pm 0.04$, HC unaffected $0.94 \pm 0.06$; Tukey HSD, $p=0.001)$. Although the mean VOR gains from the unaffected PC $(0.79 \pm$ 0.06 ) were less than the horizontal canals, they were not significantly different.

After SCD plugging, the mean percent asymmetry differences in VOR gain were $3.4 \pm 2.0 \%$ for the horizontal canal, $1.4 \pm 8.6 \%$ for the posterior canal, and $30 \pm 11.2 \%$ for the SC. The VOR gain in the plugged $\mathrm{SC}$ was significantly lower than the VOR gains in the nonplugged canals (Tukey HSD, $p<0.05$ ).

The ipsilesional VOR head-thrust gain values for each canal in the UVD subjects were significantly lower than the contralesional VOR gains (one-way paired $t$ test, $p=5.1 \times 10^{-5}$; Fig. 5). The combined mean asymmetry in VOR gain between ipsi- and contralesional head thrusts was $63 \pm 18.9 \%$ for the horizontal canal, $56 \pm 12.8 \%$ for the posterior canal, and $43 \pm 5.6 \%$ for the SC.

There was no difference in VOR gain across canals in the individuals with normal SCC function 
(ANOVA, $F=2.3, p=0.088$ ). As a group, the combined mean asymmetry in 3-D VOR gain between rightward and leftward head thrusts in normal subjects was $5.4 \pm 6.1 \%$ for the horizontal canal, $5.5 \pm 3.1 \%$ for the posterior canals, and $4.2 \pm 2.3 \%$ for the SCs.

\section{DISCUSSION}

We have shown that the DVA test applied using transient, unpredictable head thrusts in the direction of maximum sensitivity for individual SCCs-which we refer to here as the "head-thrust DVA" test or htDVA-can quantify the function of individual canals and yield results that inversely correlate with individual canal gains measured using the headthrust test and 3-D scleral coils. Even in the case of surgical occlusion in one SCC, which has been shown to have a partial response recovery (Lasker et al. 1999; Rabbit et al. 1999), we found abnormal htDVA scores. Whereas the 3-D scleral coil technique remains the gold standard for direct measurement of 3-D VOR gains from eye and head movements, htDVA is much easier and faster to perform in a clinical setting, requires less equipment and expertise than does scleral coil testing, and does not require placement of wired contacts on a patient's sclerae. Consequently, the ability of the htDVA test applied in canal planes to provide data predictive of 3-D coil VOR gain measurements suggests a role for htDVA in the clinical setting.

When the examiner's goal is to quantify function of individual SCC, DVA should be tested using transient, unpredictable, SCC-plane head thrusts instead of the active head rotation paradigm traditionally used in DVA testing. Unpredictable head rotation paradigms better isolate and measure peripheral vestibular function and are less likely to allow influences such as central preprogramming or efference copy of the motor command to assist in gaze stability (Herdman et al. 2001). Enhancement of gaze stability with predictable head movement is believed to be caused by mechanisms such as centrally preprogrammed eye rotations that boost the gain (eye velocity/head velocity) of the VOR compared with unpredictable head rotations (Jell et al. 1988; Demer et al. 1993; Hoshowsky et al. 1994; Herdman et al. 2001; Della Santina et al. 2002; Tian et al. 2002; Collewijn et al. 1983) or an efference copy of the motor command that serves to mediate successive eye rotations (Kasai and Zee 1978). Because these other mechanisms can affect gaze stability when subjects can predict or control the head rotation stimulus, using unpredictable head rotations is important in application of the htDVA test.
Conversely, when the examiner's goal is to assay the functionality of the VOR system during head velocities that reflect daily life, traditional DVA using self-generated head movements may provide a more comprehensive measure of behavior because predictive head rotations may recruit non-VOR gaze-stabilizing mechanisms (Della Santina et al., 2002; Schubert et al. 2002b).

To the extent that htDVA does not precisely reflect VOR gains measured using 3-D scleral coils and the head-thrust test, the discrepancy may indicate a useful difference between these two paradigms that can be exploited in investigations of vestibular compensation and rehabilitation paradigms. Whereas the head-thrust test VOR gains give insight into the function of individual SCCs (in subjects with normal ocular motor function), the htDVA test scores may also be affected by any behavior that augments, decreases, or supplants slow-velocity eye rotation during head rotation. Thus, improvement in htDVA scores while head-thrust VOR gains remain stable suggests that a subject is learning to enlist nonvestibular strategies for compensation.

In nonsurgically lesioned canals, we found that canal plane htDVA scores were similar, and that any htDVA score greater than 0.158 was outside of normal htDVA scores. We recommend using this cutoff as the criterion reference point for abnormal htDVA. The similarity in htDVA score across canals is in contrast to the difference between vertical and horizontal canal plane VOR gains during passive headthrust testing (Cremer et al. 1998). One possible explanation for the difference is that the htDVA test allows subjects multiple opportunities to identify the optotype with multiple trials for each acuity level. It may therefore be possible that the brain "adapts" to the htDVA test-in the form of recruiting a nonvestibular eye movement to assist gaze stability. Two nonvestibular eye rotations that may assist gaze stability during the htDVA test include smooth pursuit and saccades (Schubert et al. 2002b; Bockisch et al. 2004). We do not believe, however, that this is likely because our paradigm involves displaying optotypes only while the head is turning at velocities (120$180^{\circ} / \mathrm{s}$ ) outside the typical range of smooth pursuit $\left(<100^{\circ} / \mathrm{s}\right)$ with an optotype display time $(\sim 85 \mathrm{~ms})$ less than the latency from either a smooth pursuit eye rotation or an express saccade $(\sim 100 \mathrm{~ms}$; Fischer 1986; Krauzlis and Lisberger 1994; Krauzlis and Miles 1996). One way to identify whether subjects employ nonvestibular eye movements, such as saccades, during the htDVA task is to record eye movements using 3-D coils during canal plane htDVA testing. We plan to more definitively correlate behavior (htDVA score) with physiology (VOR gain, type of eye rotation). 
One reason that the htDVA did not precisely reflect VOR gains measured using 3-D scleral coil and head-thrust testing may be related to the contribution of the torsional (roll) VOR for determining a subject's htDVA score. Although it is well established that the torsional VOR gain is $\sim 30 \%$ lower than the pitch or yaw VOR component (Aw et al. 1996), it is believed that visual acuity is not impaired when rotating about the naso-occipital axis because the visual target remains on the fovea.

Two limitations of our study involved not recording the individual's best visual acuity and the lack of diversity in age of normal controls. We developed our paradigm such that the htDVA test ended whenever the subject reached a LogMAR score of 0.000 , equivalent to a Snellen rating of $20 / 20_{-0}$. However, many individuals have a vision better than 20/20. Although we recognize that the inclusion of a subject's best visual acuity more accurately reflects their true dynamic-versus-static change in visual acuity, this difference in score determination could not have affected the outcome of our study except by increasing the difference between static and DVA. Because age has previously been shown to affect DVA, we plan to extend the present study to investigate the effect of age on htDVA and VOR gain (Schubert et al. 2002a).

\section{CONCLUSION}

The htDVA test, which does not require scleral coil placement, magnetic field coils, or expensive oculography equipment, can identify abnormal function within an individual SCC when applied using transient, unpredictable head thrusts in the direction of maximum sensitivity of each SCC. Our data suggest that htDVA scores inversely correlate with VOR gains measured using the head-thrust test and 3-D scleral coils. Differences between these two tests might be exploited to identify and to develop rehabilitation paradigms that enhance use of nonvestibular gazestabilization strategies augmenting a deficient VOR. Whereas htDVA should not replace 3-D coils testing as an assay of individual SCC function, we propose that the htDVA test should be incorporated into the current arsenal of diagnostic tests employed in clinical diagnosis of SCG hypofunction.

\section{ACKNOWLEDGMENTS}

The authors thank John Carey, MD, Nancy Beth Smith, PA, and Amir Allak, BS, for assistance during the data collection. M.C.S. was supported by a grant from the Foundation for Physical Therapy from the American Physical Therapy Association and by K23-007926 from the
National Institute on Deafness and Other Communication Disorders (NIDCD). A.A.M. was supported by NIDCD R03DC-007346. C.C.D.S. was supported by NIDCD K08DC6216.

\section{REFERENCES}

Aw ST, Haslwanter T, Halmagy GM, Curthoys IS, Yavor RA, Todd MJ. Three-dimensional vector analysis of the human vestibuloocular reflex in response to high-acceleration head rotations. I. Responses in normal subjects. J. Neurophysiol. 76:4009-4020, 1996.

Bockisch CJ, Straumann D, Hess K, Haslwanter T. Enhanced smooth pursuit eye movements in patients with bilateral vestibular deficits. NeuroReport 15(17):2617-2620, Dec. 3, 2004.

Cremer PD, Halmagy GM, Aw ST, Curthoys IS, McGarvie LA, Todd MJ, Black RA, Hannigan IP. Semicircular canal plane head impulses detect absent function of individual semicircular canals. Brain 121 (Pt 4):699-716, 1998.

Collewijn H, Martins AJ, Steiman RM. Compensatory eye movements during active and passive head movements: fast adaptations to changes in visual magnification. J Physiol 340:259-286, 1983.

Della Santina CC, Cremer PD, Carey JP, Minor LB. Comparison of head thrust test with head autorotation test reveals that the vestibulo-ocular reflex is enhanced during voluntary head movements. Arch. Otolaryngol. Head Neck Surg. 128(9): 1044-1054, Sept., 2002.

Della Santina CC, Potyagaylo V, Migliaccio Aa, Minor LB, Carey JP. Orientation of human semicircular canals measured by three-dimensional multiplanar CT reconstruction. J. Assoc. Res. Otolaryngol. 1-16, online Aug. 9, 2005.

Demer JL, OAS JG, BaLOH RW. Visual vestibular interaction in humans during active and passive vertical head movement. J Vestib Res 3:101-114, 1993.

EwaLd JR. Physiologische Untersuchingern Uber das Endorgan des Nervus Octavus. Bergmann, Wiesbaden, (1892).

Ferris FL, Kassoff A, Bresnick GH, Bailey I. New visual acuity charts for clinical research. Am. J. Ophthalmol. 94:91-96, 1982.

Fischer B. Express saccades in man and monkey. Prog. Brain Res. 64:155-160, 1986.

Halmagy GM, Curthoys IS. A clinical sign of canal paresis. Arch. Neurol. 45(7):737-739, 1988.

HASLWANTER T. Mathematics of three-dimensional eye rotations. Vis. Res. 35:1727-1739, 1995.

Hepr K. On listing's law. Commun. Math. Phys. 132:285-295, 1990.

Herdman SJ, Tusa RJ, Blatt P, Suzuki A, Venuto PJ, Roberts D. Computerized dynamic visual acuity test in the assessment of vestibular deficits. Am. J. Otol. 19(6):790-796, 1998.

Herdman SJ, Schubert MC, Tusa RJ. Role of central preprogramming in dynamic visual acuity with vestibular loss. Arch. Otolaryngol. Head Neck Surg. 127(10):1205-1210, Oct., 2001.

Herdman SJ, Schubert MC, Das VE, Tusa RJ. Recovery of dynamic visual acuity in unilateral vestibular hypofunction. Arch. Otolaryngol. Head Neck Surg. 129(8):819-824, 2003.

Hoshowsky B, Tomlinson D, Nedzelski J. The horizontal vestibuloocular reflex gain during active and passive high frequency head movements. Laryngoscope 104:140-145, 1994.

Jell RM, Stockwell CW, Turnipseed GT, et al. The influence of active versus passive head oscillation, and mental set on the human vestibulo-ocular reflex. Aviat Space Environ Med 59:1061-1065, 1988.

KasaI T, Zee DS. Eye-head coordination in labyrinthine-defective human beings. Brain Res. 144(1):123-141, Apr 7, 1978. 
Krauzlis RJ, Lisberger SG. Temporal properties of visual motion signals for the initiation of smooth pursuit eye movements in monkeys. J. Neurophysiol. 72:150-162, 1994.

Krauzlis RJ, Miles FA. Release of fixation for pursuit and saccades in humans: evidence for shared inputs acting on different neural substrates. J. Neurophysiol. 76:2822-2833, 1996.

Lasker DM, Backous DD, Lysakowski A, Davis GL, Minor LB. Horizontal vestibuloocular reflex evoked by high-acceleration rotations in the squirrel monkey. II. Responses after canal plugging. J. Neurophysiol. 82:1271-1285, Sep, 1999.

Migliaccio AA, Todd MJ. Real-time rotation vectors. Australas. Phys. Eng. Sci. Med. 22:73-80, 1999.

Migliaccio AA, Minor LB, CARey JP. Vergence-mediated modulation of the human horizontal vestibulo-ocular reflex is eliminated by a partial peripheral gentamicin lesion. Exp. Brain Res. 159(1): 92-98, Nov., Epub Jun. 24, 2004.
MiNOR LB. Clinical manifestations of superior semicircular canal dehiscence. Laryngoscope 115(10):1717-1727, Oct., 2005.

RABbit RD, Boyle R, Highstein SM. Influence of surgical plugging on horizontal semicircular canal mechanics and afferent response dynamics. J Neurophysiol 82(4):1033-1053, Aug., 1999.

Schubert MC, Herdman SJ, Tusa RJ. Vertical dynamic visual acuity in normal subjects and patients with vestibular hypofunction. Otol. Neurotol. 23(3):372-377, 2002a.

Schubert MC, Das VE, Tusa RJ, Herdman SJ. Gaze stability during predictable and unpredictable head thrusts. Program No. 266.1. Washington, DC: Society for Neuroscience. CD-ROM, (2002b)

Tian JR, Shubayev I, Demer JL. Dynamic visual acuity during passive and self-generated transient head rotation in normal and unilaterally vestibulopathic humans. Exp Brain Res 142(4):486-495, Feb., 2002. Epub Dec. 14, 2001. 